\title{
Novel mixed potential sensor device to compare two gas compartments and to determine directly the conversion of an automotive catalyst
}

\author{
Thomas Ritter, Gunter Hagen, Ralf Moos \\ Bayreuth Engine Research Center, Department of Functional Materials, University of Bayreuth, \\ Universitätsstraße 30, 95440 Bayreuth, Germany \\ funktionsmaterialien@uni-bayreuth.de
}

\begin{abstract}
:
In this work, a novel sensor device is developed. It enables to compare two gas compartments electrochemically by applying the mixed potential sensing principle. Thereby, disadvantages that come along with the usually-used half-cell setups like indirect heating, complicated manufacturing and only destructive dismantling of sample and sealing are overcome. The device bases on an yttria-stabilized zirconia disc that is equipped with an integrated heater to provide a hot area in the center for sensing while at the same time it is cold enough at the outer radius for contacting with a polymer sealing $(\mathrm{O}$ ring). As an example for its functionality, the gas mixtures up- and downstream of a catalyst were compared, while the sensor voltage correlates well with catalyst conversion.
\end{abstract}

Key words: compare two gas compartments electrochemically, mixed potential sensor, yttriastabilized zirconia disc, catalyst conversion, on-board diagnosis

\section{Introduction}

Determining the content of certain gas components in automotive exhaust is not possible without gas sensors that provide longterm stability regarding the raw atmospheres. Therefore, commonly used systems base on zirconia solid state electrolytes because of their high mechanical strength and chemical stability [1]. An intensive investigated field of such systems is the mixed potential sensing principle, since it enables selective gas component detection [2-7].

Solid state mixed potential sensors are often characterized by using half-cell setups $[8,9]$. There, the sensor signal is determined by exposing one side of the device to a reference atmosphere and the other side to the gas mixture to be analyzed. Such setups often require indirect heating and a complicated manufacturing process, while often the dismantling of the sensor sample and the sealing is destructive.

Hence, in this work a self-heated sensor setup is developed. It ensures that the device is in its center hot enough for sufficient ionic conductivity and electrode activity, while it has also cold areas for gas tight plastic sealings, e.g., with rubber O-rings. Then the device can be installed in a housing that provides all the necessary electrical connections.

\section{Sensor layout}

Fig. 1 shows a scheme of the sensor device that bases on an yttria-stabilized zirconia (YSZ) disc. An electrode is placed on each side of the device whereas its supply line is insulated to the ambient as well as to the YSZ to ensure that the center of the disc is connected ionically. There, an integrated heater meanders around the electrodes buried in the middle of the disc (for an equal temperature at both electrodes) and embedded in an insulation layer to suppress electrochemical blackening. To

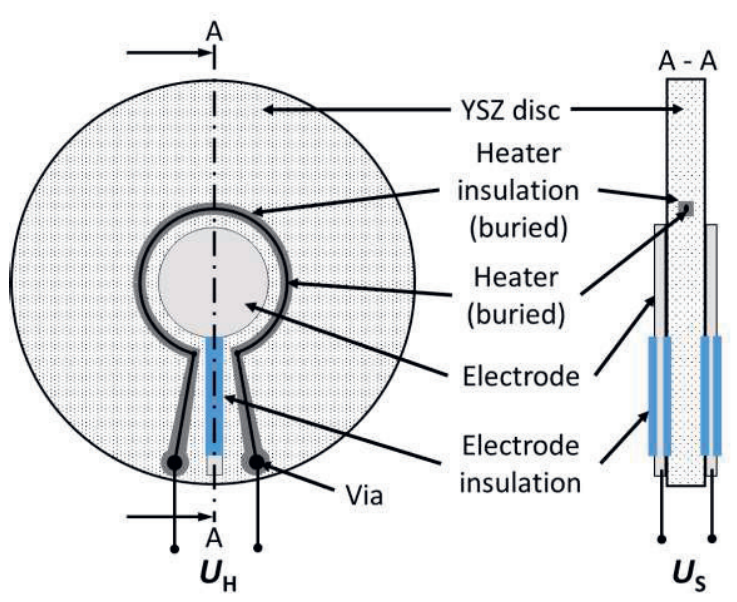

Fig. 1. Scheme of the sensor device layout. 
realize this design, the sensor device is manufactured in high temperature co-fired ceramics (HTCC) technology. On choosing a suitable disc diameter it is possible to reach high enough temperatures (up to $550{ }^{\circ} \mathrm{C}$ ) at the electrodes while the circumference of the device remains cold enough (below $200^{\circ} \mathrm{C}$ ) due to the low thermal conductivity of YSZ. Since a high temperature gradient along the radius of the device leads to high thermal stress and consequently a damaged device, finite element simulations were conducted to find suitable geometric dimensions regarding a cold enough outer radius while thermal stress is reduced to a minimum. Therefore, a model analogous to the layout shown in Fig. 1 was set up in COMSOL Multiphysics ${ }^{\circledR}$. Several geometry parameters were varied to understand how they affect the temperature distribution until an appropriate sensor design was found.

The manufactured devices according to the described layout obtained a highly axisymmetric temperature distribution so they could be arranged for measurements as shown in Fig. 2. To provide two cells that can be flooded with gas, two cylinders made for example from polyether ether ketone (PEEK) or glass and a polymer sealing (O-ring) were pressed on each side of the sensor device.

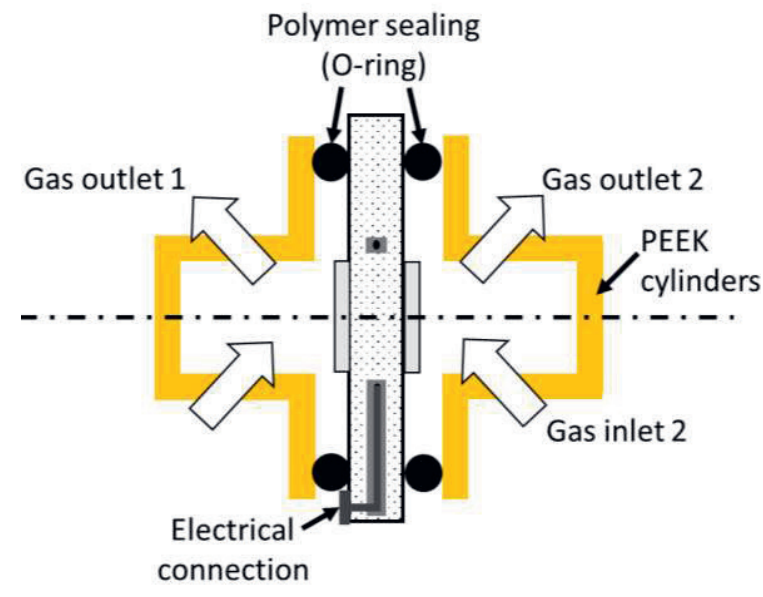

Fig. 2: Scheme of setup used for measurements.

\section{Experimental results}

To validate its functionality for comparing electrochemically two gas mixtures based on the mixed potential sensing principle, the sensor devices were used to determine the conversion of an oxidation catalyst according to $[10,11]$ with the setup shown in Fig. 3 .

Therefore, the conversion of a catalyst is simulated here by dosing different hydrocarbon

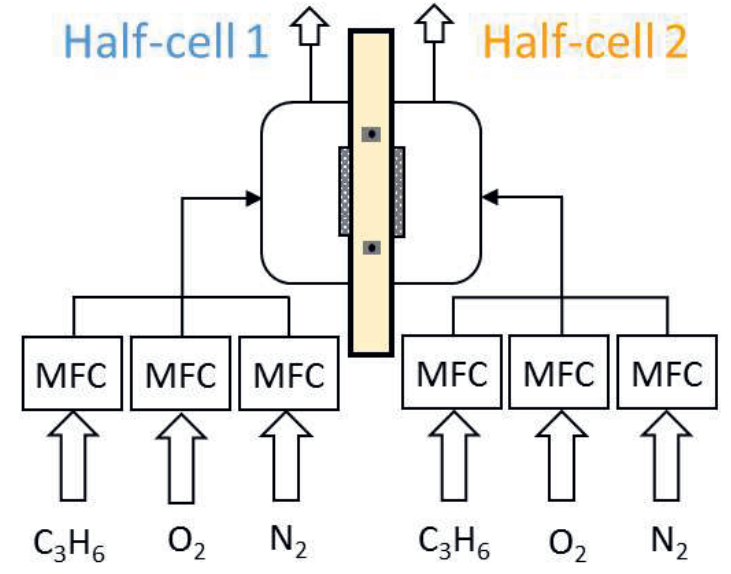

Fig. 3: Scheme of the setup used to compare two gas compartments.

concentrations in the two half-cells of the sensor setup as it is shown in the upper graph in Fig. 4 where the gas concentration of the two half-cells is shown over time measured by a flame ionization detector (FID). There is a constant propene concentration in half-cell 1 , which should represent the gas mixture upstream of the catalyst. The propene content in half-cell 2 is reduced step-wise as it should simulate an increased catalyst activity and the corresponding increased propene conversion, since it represents a gas mixture downstream of a catalyst. Then, the "synthetic" propene conversion can be calculated assuming the propene content in half-cell 1 is the feed concentration of the catalyst. The conversion is shown in the middle graph in Fig. 4.

The lower graph in Fig. 4 shows the measured sensor signal on setting the sensor temperature to $500{ }^{\circ} \mathrm{C}$. For the first $1000 \mathrm{~s}$, when the propene content in the half-cell is almost the same, the sensor signal is also near $0 \mathrm{mV}$ since there is theoretically no difference between the two gas compartments. As it can be seen, when the propene concentration is reduced the sensor signal correlates well with the increased propene conversion, while the measured signal is stable and fast responding. When the propene concentration is step-wise brought back to the "feed"-concentration, there is almost no hysteresis regarding the sensor signal on reducing the propene content in half-cell 2 .

It has to be mentioned that the sensor signal is slightly influenced by the applied heater voltage. Heating with ac voltage reduces this influence. The curve in the lower graph in Fig. 4 are raw data and the noise is negligible. 


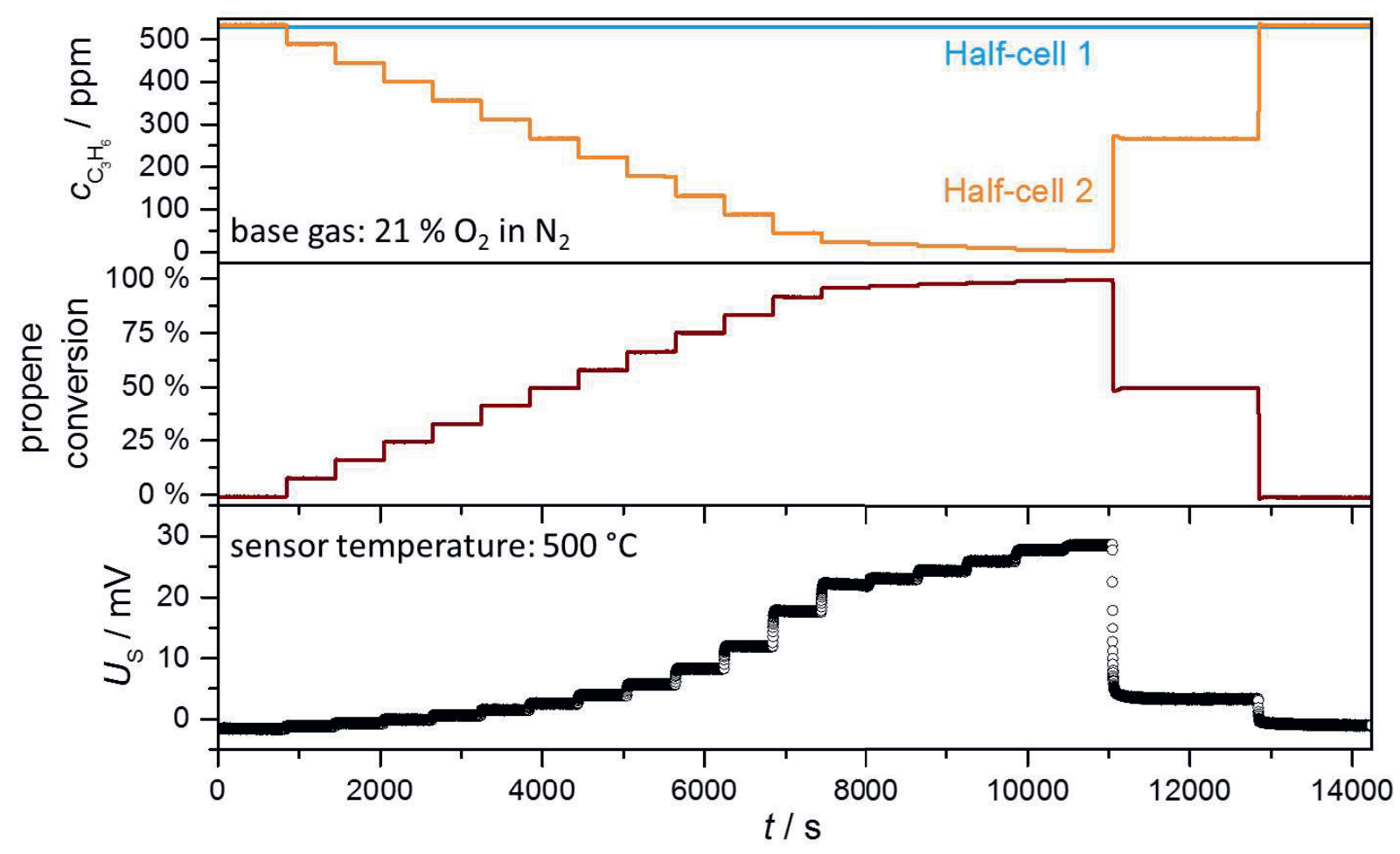

Fig.4. Measurement on comparing two gas mixtures electrochemically with a simulated, „synthetic" conversion of propene. Upper graph: Propene concentration in the two half-cells over time measured by flame ionization detector (FID). Middle graph: Calculated "synthetic" propene conversion from FID data. Lower graph: Sensor signal over time.

\section{Conclusion and outlook}

The presented device is able to replace common solid state mixed potential sensor setups, as it provides high temperatures for sufficient ionic conductivity and cold areas for low temperature sealing. It is possible to perform fast measurement series regarding comparison of mixed potential electrode configurations since exchanging and producing of the device is uncomplicated.

Measurements show fast sensor signal response and stable signals only slightly influenced by the integrated heater. It is also suitable for the presented function as a conversion sensor, while further investigations will focus on proving the theory that the sensor signal does not depend on the feed gas concentration but only on catalyst conversion. Regarding common mixed potential investigations we will also reproduce measurements known from literature with the new device, where the sensing electrode faces a gas mixture with a specific analyte while the reference electrode is exposed to wet air or similar.

\section{References}

[1] J. Riegel: Exhaust gas sensors for automotive emission control, Solid State lonics, 152-153, 783-800 (2002), doi: 10.1016/S01672738(02)00329-6
[2] J.W. Fergus: Sensing mechanism of nonequilibrium solid-electrolyte-based chemical sensors, Journal of Solid State Electrochemistry, 15, 971-984 (2011), doi: 10.1007/s10008-0101046-4

[3] G. Lu, N. Miura, N. Yamazoe: Stabilized zirconia-based sensors using $\mathrm{WO}_{3}$ electrode for detection of $\mathrm{NO}$ or $\mathrm{NO}_{2}$, Sensors and Actuators B: Chemical, 65, 125-127 (2000), doi: 10.1016/S0925-4005(99)00398-6

[4] R. Mukundan, E. L. Brosha, D. R. Brown, and F. H. Garzon: A Mixed-Potential Sensor Based on a $\mathrm{Ce}_{0.8} \mathrm{Gd}_{0.2} \mathrm{O}_{1.9}$ Electrolyte and Platinum and Gold Electrodes, Journal of The Electrochemical Society, 147, 1583-1588 (2000), doi: 10.1149/1.1393398

[5] D. Schönauer-Kamin, M. Fleischer, R. Moos: Half-cell potential analysis of an ammonia sensor with the electrochemical cell $\mathrm{Au}|\mathrm{YSZ}| \mathrm{Au}$, $\mathrm{V}_{2} \mathrm{O}_{5}-\mathrm{WO}_{3}-\mathrm{TiO}_{2}$, Sensors, 13, 4760-4780 (2013), doi: 10.3390/s130404760

[6] J. Zosel, G. Schiffel, F. Gerlach, K. Ahlborn, U. Sasum, V. Vashook, U. Guth: Electrode materials for potentiometric hydrogen sensors, Solid State lonics, 177, 2301-2304 (2006), doi: 10.1016/j.ssi.2006.01.004

[7] N. Miura, T. Raisen, G. Lu, N. Yamazoe: Highly selective $\mathrm{CO}$ sensor using stabilized zirconia and a couple of oxide electrodes, Sensors and Actuators B: Chemical, 47, 84-91 (1998), doi: 10.1016/S0925-4005(98)00053-7

[8] N. Miura, T. Sato, S.A. Anggraini, H. Ikeda, S. Zhuiykov: A review of mixed-potential type zirconia-based gas sensors, lonics, 20, 901-925 (2014), doi: 10.1007/s11581-014-1140-1 
[9] J. Zosel, D. Westphal, S. Jakobs, R. Müller, U. Guth: Au-oxide composites as HC-sensitive electrode material for mixed potential gas sensors, Solid State Ionics, 152-153, 525-529 (2002), doi: 10.1016/S0167-2738(02)00355-7

[10] G. Hagen, K. Burger, S. Wiegärtner, D. Schönauer-Kamin, R. Moos: A mixed potential based sensor that measures directly catalyst conversion-A novel approach for catalyst onboard diagnostics, Sensors and Actuators B: Chemical, 217, 158-164 (2015), doi: 10.1016/j.snb.2014.10.004

[11] T. Ritter, G. Hagen, J. Kita, S. Wiegärtner, F. Schubert, R. Moos: Self-heated HTCC-based ceramic disc for mixed potential sensors and for direct conversion sensors for automotive catalysts, Sensors and Actuators B: Chemical, in press (2016), doi: 10.1016/j.snb.2016.11.079 